\title{
SNR degradation in GNSS-R measurements under the effects of Radio-Frequency Interference
}

\author{
Jorge Querol, Student Member, IEEE, Alberto Alonso-Arroyo, Student Member, IEEE, \\ Raul Onrubia, Student Member, IEEE, Daniel Pascual, Student Member, IEEE, Huyk Park, Member, IEEE, \\ and Adriano Camps, Fellow, IEEE
}

\begin{abstract}
Radio-Frequency Interference (RFI) is a serious threat for systems working with low power signals such as those coming from the Global Navigation Satellite Systems (GNSS). The Spectral Separation Coefficient (SSC) is the standard figure of merit to evaluate the Signal-to-Noise Ratio (SNR) degradation due to RFI. However, an in-depth assessment in the field of GNSS-Reflectometry (GNSS-R) has not been performed yet, and particularly, about which is the influence of RFI on the so-called Delay-Doppler Map (DDM). This work develops a model that evaluates the contribution of intra-/inter-GNSS and external RFI effects to the degradation of the SNR in the DDM for both conventional and interferometric GNSS-R techniques. Moreover, a Generalized SSC (GSSC) is defined to account for the effects of non-stationary RFI signals. The results show that highly directive antennas are necessary to avoid interference from other GNSS satellites, whereas mitigation techniques are essential to keep GNSS-R instruments working under external RFI degradation.
\end{abstract}

Index Terms-RFI, interference, GNSS-R, reflectometry, SNR, DDM, degradation, WAF, ambiguity, SSC, GSSC, Wigner-Ville.

\section{INTRODUCTION}

$\mathbf{N}$ OWADAYS, the consolidation of mass-market wireless communication applications together with the increasing demand of wider bandwidths of operation have fostered the problem of Radio-Frequency Interference (RFI) in an overcrowded frequency spectrum. RFI are undesirable signals that degrade or even disrupt the performance of a receiver, and they are particularly threatening for devices that use very low power signals such as Global Navigation Satellite Systems (GNSS) receivers. Despite their inherent protection to RFI due to the spread-spectrum codes used in navigation signals in order to achieve Code Division Multiple Access (CDMA), GNSS-based instruments are prone to suffer from RFI effects. In the last years, the number of reported RFI occurrences in GNSS has been increasing [1], even though legal policies are established to protect GNSS bands. Furthermore, several studies, such as [2] and [3], have shown that, in addition to jamming signals, even unintentional RFI signals can degrade the performance of GNSS receivers. In GNSS, RFI signals include aeronavigation signals such as Distance Measurement Equipment (DME) at the GPS L5/Galileo E5 bands, spurious or harmonics of lower frequency, and even contributions of

This work has received funding by the project "AGORA: Técnicas Avanzadas en Teledetección Aplicada Usando Señales GNSS y Otras Señales de Oportunidad" of the Spanish Ministerio de Economía y Competitividad (MINECO/FEDER) ESP2015-70014-C2-1-R, and by the grant for the recruitment of early-stage research staff FI-DGR 2016 of the AGAUR/Generalitat de Catalunya 2016FI-B00738. the same GNSS satellites sharing the same band of operation among others. Although anthropogenic RFI signals are the most common source, natural emissions may also be considered as RFI signals such as Sun L-band surface glints which can also affect GNSS-R measurements.

The impact of RFI signal in GNSS receivers has been studied in detail over the last decade. The Spectral Separation Coefficient (SSC) was defined as a figure of merit to quantify the degree of interference that a GNSS signal suffers due to other signals sharing the band of operation [4], in terms of a reduction of the Signal-to-Noise Ratio (SNR). Indeed, the SSC allows to characterize also GNSS intra-system and intersystem SNR degradation [5]. That is a measurement of the rejection ratio between navigation codes taken into account in their design process in order to guarantee the co-existence of multiple GNSS.

The RFI problem in GNSS is of special concern due to the high number of existing GNSS-enabled applications. One of these applications is GNSS-Reflectometry (GNSS-R), a promising technique in the field of remote sensing, that was first proposed in 1988 for scatterometry applications [6]. Later in 1993, GNSS-R was first suggested for mesoscale ocean altimetry [7], and since then, this technique has been used in the retrieval of many geophysical parameters such as measuring sea surface state [8], sea surface salinity [9], soil moisture [10], and ocean altimetry [11] among others.

GNSS-R devices receive simultaneously the signals transmitted by multiple GNSS satellites that have been scattered by the surface of interest. This configuration is known as multistatic in radar theory [12]. There are two main GNSS-R approaches [13]. The first is the so-called conventional GNSS$\mathrm{R}$ (cGNSS-R), in which the reflected signal is cross-correlated with a locally generated replica of the transmitted one. Only signals with open codes can be used in this approach (e.g. GPS L1 C/A). The second one is the so-called interferometric GNSS-R (iGNSS-R), in which the reflected signal is crosscorrelated with a signal captured directly from the satellite. In this case, both open and restricted codes are used simultaneously in a specific band (e.g. C/A, C, P and M codes at GPS L1 band). Figure 1 illustrates the scenario corresponding to each approach. Regarding their resilience in front of RFI, cGNSS-R is not immune to RFI due to the finite rejection ratio of the spread-spectrum signal, whereas iGNSS-R is even more prone to RFI, since an undesired signal captured by both antennas will produce a non-zero cross-correlation.

The observable that contains all the information of GNSS- 


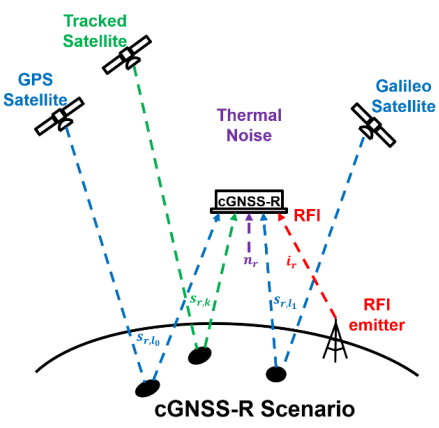

(a)

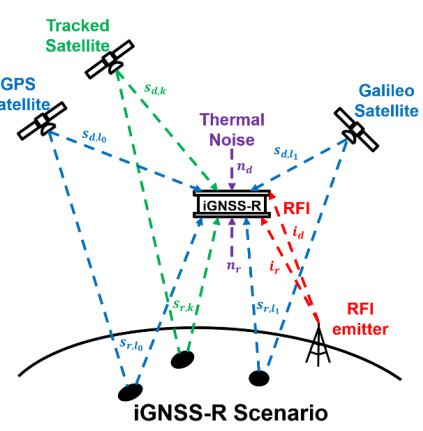

(b)
Fig. 1: Illustrative diagram representing satellite, RFI and thermal noise signals involved in the study of the SNR degradation for a) cGNSS-R scenario, and b) iGNSS-R scenario.

$\mathrm{R}$ measurements is the so-called Delay-Doppler Map (DDM), which shows how the scattering process over the surface of interest spreads the energy of the transmitted signal in the delay-Doppler space, and how this spreading changes in time. The generally accepted model for incoherent GNSS$\mathrm{R}$ reflections, assuming fully diffuse scattering from rough surfaces, and originally stated for sea-surface observations, is the Zavorotny-Voronovich (Z-V) model [14]. The Z-V model can be expressed in a simplified version as a two dimensional convolution [15], and is equivalent to the so-called radar mapping equation [12]. The model can be expressed as in [16] as:

$$
\left\langle|Y(\nu, \tau)|^{2}\right\rangle=|\chi(\nu, \tau)|^{2} * * \Sigma(\nu, \tau),
$$

where $\left\langle|Y(\nu, \tau)|^{2}\right\rangle$ is the expected DDM, $\nu$ and $\tau$ are the Doppler and delay variables respectively, the $* *$ operator indicates a two-dimensional convolution in $\nu$ and $\tau$ domains, $|\chi(\nu, \tau)|^{2}$ is the Woodward's Ambiguity Function (WAF) of the navigation code, and $\Sigma(\nu, \tau)$ accounts for the scattering at the surface, propagation losses, antenna patterns, and the rest of terms in the bistatic radar equation. Moreover, if the reflection process is coherent because it takes place over a planar surface (e.g. calm water, ice floes, or flat lands), the measured DDM will be an attenuated version of the signal WAF. Furthermore, any combination of coherent and incoherent reflection will led to a DDM that can be expressed as an attenuated and distorted version of the signal WAF.

The WAF is the squared modulus of the ambiguity function first proposed in [17], and it was introduced in radar theory [18] as the appropriate function to evaluate the degree of ambiguity in the measurements when the number of radar echoes is sufficiently large. This approach is translated in a non-coherent detection process after the cross-correlation as used in most of GNSS receivers [19] in order to increase the SNR, and necessary in GNSS-R applications due to the effect of speckle noise introduced in the scattering process [12], which is caused by the addition of a large number of random phased scatterers over the surface of interest.

According to the experiments carried out in [2], the effect of RFI signals in cGNSS-R is translated into an equivalent rise of the noise floor, and thus, into a desensitization of the receiver. However, these effects have not been studied in depth to the best of authors' knowledge. This work aims at providing a general model that accounts for the effects of RFI signals in GNSS-R instruments operating with CDMA spread-spectrum GNSS signals, including intra- and inter-GNSS interference and other external RFI signals.

The results derived along this work are completely general, so they can be applied to any GNSS-R scenario using the proper geometry parameters. However, for the sake of clarity, these results are illustrated using specific cases that take into account no spatial filtering (isotropic antennas), perfect specular reflection (no scattering), and equal received power for all satellites (same transmission power, propagation attenuation, and surface reflectivity).

The outline of this paper is as follows. In Section II, the general model of SNR degradation is developed for both cGNSS-R and iGNSS-R techniques. In Section III and Section IV, the particular effects on the SNR of cross-correlation between GNSS signals, and external RFI signals, respectively, are described in detail. The main conclusions of this paper are stated in Section V.

\section{GENERAL SNR MODEL FOR GNSS-R}

\section{A. Signal model and detection}

After the scattering process, GNSS-R signals are collected by the instrument antennas, conditioned by the front-end stage with bandwidth $B_{r}$, and finally delivered to the processing stage. The complex base-band signal at this stage can be expressed as the addition of three terms:

$$
y_{r}(t)=s_{r}(t)+i_{r}(t)+n_{r}(t)=\sum_{l=0}^{L-1} s_{r_{l}}(t)+i_{r}(t)+n_{r}(t)
$$

where the subscript $r$ stands for "reflected" signals, $L$ is the number of "in-view" GNSS signals, $s_{r_{l}}$ corresponds to the lth signal, $i_{r}$ is the aggregate of RFI signals, and $n_{r}$ is bandlimited thermal noise (see Fig. 1a).

GNSS signals are detected after the delay-Doppler correlator or "matched filter" that performs the spread-spectrum demodulation [19]. In cGNSS-R, the signal after the correlator for a particular $k$ th tracked signal (e.g., GPS L1 C/A, Galileo E5, Beidou B2) is obtained as:

$$
\chi_{y_{r} c_{k}}(\nu, \tau)=\frac{1}{T_{c}} \int_{T_{c}} y_{r}(t) c_{k}^{*}(t-\tau) e^{-j 2 \pi \nu t} d t,
$$

where $y_{r}(t)$ is the received complex base-band signal defined in (2); $c_{k} \in \mathbb{C}$ is the clean replica of the $k$ th navigation code, assumed known, deterministic and with normalized power within the bandwidth $B_{r} ; \nu$ and $\tau$ are the Doppler and delay variables respectively; and $T_{c}$ is known as the coherent integration time.

As aforementioned, the detection process in GNSS-R must be non-coherent as in radar systems, so that the new observable after the detector becomes the expected power expressed as:

$$
|\chi|_{y_{r} c_{k}}^{2}(\nu, \tau)=\mathrm{E}\left\{\left|\chi_{y_{r} c_{k}}(\nu, \tau)\right|^{2}\right\}
$$


The expected operator $\mathrm{E}\{\cdot\}$ is introduced since the signal $y_{r}$ is a random process. Furthermore, it is often replaced by the average operator $\langle\cdot\rangle$ as an unbiased estimator of the expected value, and usually referred as incoherent averaging [14]. In this work, $|\chi|_{y_{r} c_{k}}^{2}$ is introduced as the Cross-WAF (CWAF) between $y_{r}$ and $c_{k}$ analogously to the definition of the (Auto-) WAF for random processes in radar theory [18]. Then, $|\chi|_{y_{r} c_{k}}^{2}$ turns to be equal to the measured DDM for the $k$ th satellite signal using the cGNSS-R approach, and equivalent to the expected DDM $\left\langle|Y(\nu, \tau)|^{2}\right\rangle$ defined in [14]. In other words, the CWAF is the mathematical operation used to compute the measured DDM.

In iGNSS-R, the clean replica of the code $c_{k}$ is replaced by the signal acquired directly from the "in-view" satellites defined as

$$
y_{d}(t)=s_{d}(t)+i_{d}(t)+n_{d}(t)=\sum_{l=0}^{L-1} s_{d_{l}}(t)+i_{d}(t)+n_{d}(t),
$$

where the subscript $d$ stands for "direct" signals (see Fig. 1b), and it is analogous to (2). Therefore, the output of the correlator becomes $\chi_{y_{r} y_{d}}(\nu, \tau)$, and the DDM in iGNSS-R can be defined as $|\chi|_{y_{r} y_{d}}^{2}(\nu, \tau)$. Furthermore, signal $y_{d}$ is treated as a unit-less signal since it becomes the impulse response of the "matched" filter, and its power represents a non-dimensional scale factor in the iGNSS-R DDM.

\section{B. DDM decomposition}

In order to study the contribution of RFI signals to the measured DDM, the CWAF is decomposed into the terms corresponding to each signal in (2). Assuming that $s_{r}, i_{r}$, and $n_{r}$ have zero mean and are statistically independent from each other, $|\chi|_{y_{r} c_{k}}^{2}(\nu, \tau)$ can be expressed, using the Sussman's Formula [20], as follows (see proof in Appendix A):

$$
|\chi|_{y_{r} c_{k}}^{2}(\nu, \tau)=|\chi|_{s_{r, k} c_{k}}^{2}(\nu, \tau)+\sum_{\substack{l \geq 0 \\ l \neq k}}^{L-1}|\chi|_{s_{r, l} c_{k}}^{2}+|\chi|_{i_{r} c_{k}}^{2}+|\chi|_{n_{r} c_{k}}^{2}
$$

where the first term corresponds to the useful tracked signal DDM, the second one is the cross-sat term, the third one is the RFI term, and the last one is the thermal noise term. Figure 2 depicts an hypothetical example of DDM decomposition showing each one of the terms corresponding to a cGNSS-R DDM using GPS L1 C/A with $B_{r}=2.046 \mathrm{MHz}$.

The CWAF between the reflected signal of the $k$ th tracked satellite, $s_{r_{k}}$, and its clean replica, $c_{r_{k}},|\chi|_{s_{r_{, k} c_{k}}}^{2}(\nu, \tau)$, is coined as tracked or useful signal DDM in this work. The tracked signal DDM contains all the information about the surface of interest, and hence, the rest of the terms in (6) represent undesired contributions which degrade the SNR. Besides, in cGNSS-R, all of them are translated into a rise of a uniform power floor as it can be appreciated in Fig. 2. Among them, the cross-sat term include all intra- and inter-GNSS interference whereas the RFI term represents the additive contribution of external RFI signals to the received DDM. Both terms will be analyzed in detail in next sections. Eventually, assuming that the thermal noise after the front-end is a complex Gaussian

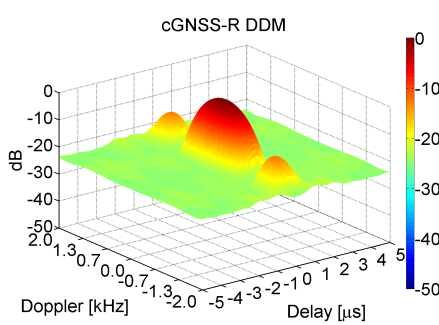

(a)

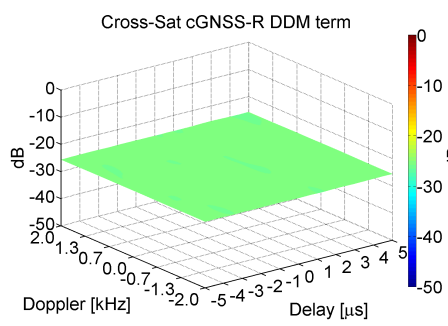

(c)

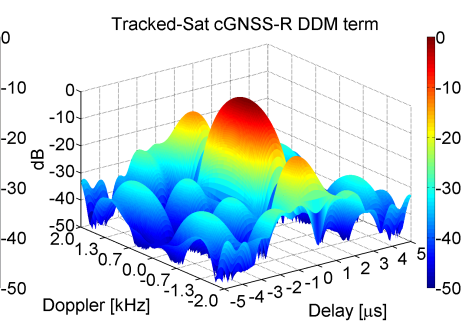

(b)

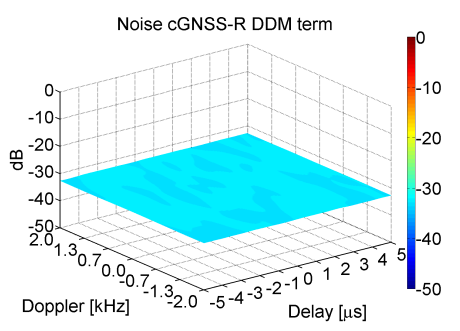

(e)

Fig. 2: Sample decomposition of a cGNSS-R DDM. The following conditions have been assumed: GPS L1 C/A code, perfect specular reflection (no scattering), $B_{r}=2.046 \mathrm{MHz}$, $T_{c}=1 \mathrm{~ms}$, and equal unitary received power for all signals. Each subfigure corresponds to (a) the received DDM obtained from (6), (b) the tracked signal DDM, (c) the cross-sat term (for 10 "in-view" additional satellites: 5 GPS L1 + 5 Galileo E1), (d) the RFI term (jammer chirp RFI), and (e) the noise term.

stochastic process, with flat Power Spectral Density (PSD) within $B_{r}$, and equal to $N_{0_{r}}$, the noise term in cGNSS-R can be approximated as:

$$
|\chi|_{n_{r} c_{k}}^{2}=\frac{N_{0_{r}}}{T_{c}} \int_{\nu-B_{r} / 2}^{\nu+B_{r} / 2} S_{c_{k}}(f) d f \simeq \frac{N_{0_{r}}}{T_{c}},
$$

where $S_{c_{k}}(f)$ is the PSD function of $c_{k}$, and it has been considered that the range of the variations of $\nu$ is very small as compared to $B_{r}$.

On the other hand, the DDM in iGNSS-R can be obtained from $|\chi|_{y_{r} y_{d}}^{2}(\nu, \tau)$. Taking into account the same assumptions as in cGNSS-R, and that $s_{d}, i_{d}$, and $n_{d}$ are statistically independent between them, the iGNSS-R DDM can be expressed as in (8) (see proof in Appendix B), where each term represents from left to right: the useful tracked signal DDM as in (6) but for a GNSS composite signal, the crosstalk term, the cross-sat term, the RFI term, and the last three ones are the interferometric thermal noise terms (i.e. $|\chi|_{\text {noise }}^{2}$ ). 


$$
|\chi|_{y_{r} y_{d}}^{2}(\nu, \tau)=|\chi|_{s_{r, k} s_{d, k}}^{2}(\nu, \tau)+|\chi|_{\text {cross-talk }}^{2}+|\chi|_{\text {cross-sat }}^{2}+|\chi|_{\mathrm{RFI}}^{2}(\nu, \tau)+\left(|\chi|_{n_{r} s_{d, k}}^{2}+|\chi|_{s_{r, k} n_{d}}^{2}+|\chi|_{n_{r} n_{d}}^{2}\right)
$$

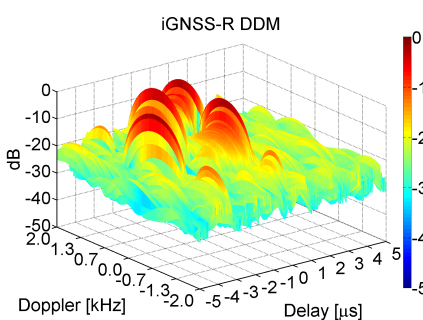

(a)

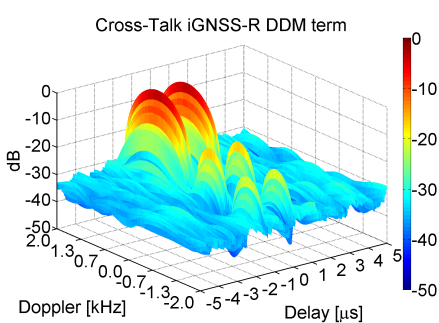

(c)

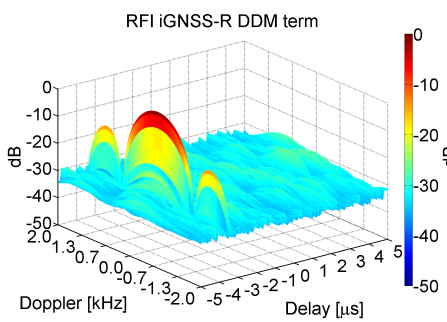

(e)

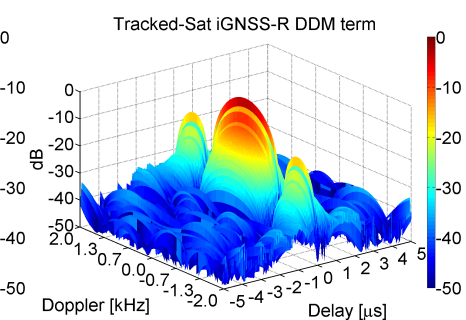

(b)

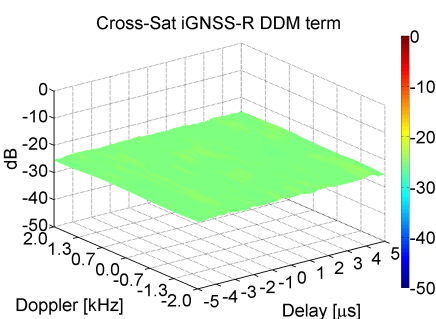

(d)

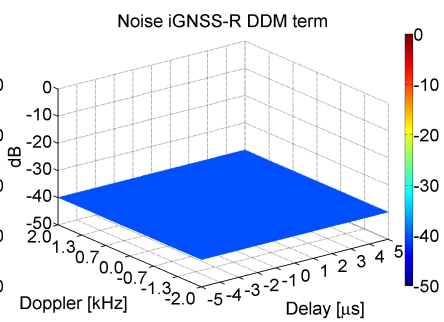

(f)
Fig. 3: Sample decomposition of a iGNSS-R DDM. The following conditions have been assumed: GPS composite L1 (C/A, $\mathrm{C}, \mathrm{P}(\mathrm{Y})$ and $\mathrm{M}$ codes), perfect specular reflection (no scattering), $B_{r}=30.69 \mathrm{MHz}, T_{c}=1 \mathrm{~ms}$, and equal unitary received power for all signals. Each subfigure corresponds to (a) the received DDM, obtained from (8), (b) the tracked signal DDM, (c) the cross-talk term (for 10 "in-view" additional satellites: 5 GPS L1 + 5 Galileo E1), (d) the cross-sat term, (e) the RFI term (jammer chirp RFI), and (f) the noise terms.

Besides, in iGNSS-R, the subscript $k$ is used to indicate all those codes that belong to a GNSS composite signal received from a particular satellite (e.g.; GPS L2 composed by C, P(Y), and $\mathrm{M}$ codes; Galileo E6 composed by CS, and PRS codes; Beidou B3 with a single code), whereas, in cGNSS-R, the subscript $k$ refers to the particular clean code replica used in the delay-Doppler correlator (e.g GPS L1 C/A).

An example showing each one of the terms corresponding to an iGNSS-R DDM, using GPS composite L1 signal with $B_{r}$ $=30.69 \mathrm{MHz}$, is depicted in Fig. 3. Note that the comparison between Figures 2 and 3 is not direct since the assumed frontend bandwidth $B_{r}$ differs in each case.

In (8), the cross-talk term appears as an exclusive effect of the iGNSS-R technique. Cross-talk is composed by the DDMs corresponding to the "in-view" non-tracked satellite signals, and it can have an overwhelming effect on the SNR (much higher than cross-sat), if it overlaps the tracked signal DDM. The overlapping between DDMs depends on the relative position and velocity between the satellites and the receiver, on the WAF of the involved signals, and on the surface of reflection. A detailed study of cross-talk effect is beyond the scope of this paper (see [21]).

Moreover, interferometric cross-sat and RFI terms are equivalent to the ones in the cGNSS-R case. Eventually, interferometric noise terms can be approximated as follows:

$$
|\chi|_{\text {noise }}^{2} \simeq \frac{N_{0_{r}}}{T_{c}} P_{s_{d, k}}+P_{s_{r, k}} \frac{N_{0_{d}}}{T_{c}}+N_{0_{r}} N_{0_{d}} \frac{B_{r}}{T_{c}},
$$

where $T_{c}$ is the coherent integration time, $N_{0_{r}}$ and $N_{0_{d}}$ are the PSD of thermal noise after the respective front-end after down-looking and up-looking antennas with equal bandwidth $B_{r}$, and considering the same approximation applied in (7). This expression of the interferometric noise matches with the one developed in [22].

\section{SNR definition and degradation}

The SNR is the figure of merit that has been chosen to evaluate the impact of RFI on GNSS-R measurements, and it is typically defined at the maximum of the tracked signal DDM. In this paper, in order to maintain the whole information of the DDM, a point-to-point relation will be considered, similarly to the one used in [22]. Therefore, a general SNR definition for GNSS-R measurements considering the $k$ th tracked satellite signal can be written as:

$\rho_{k}(\nu, \tau)=\frac{|\chi|_{k \text { th-sat }}^{2}(\nu, \tau)}{|\chi|_{\text {cross-talk }}^{2}(\nu, \tau)+|\chi|_{\text {cross-sat }}^{2}+|\chi|_{\mathrm{RFI}}^{2}(\nu, \tau)+\underset{(10)}{|\chi|_{\text {noise }}^{2}}}$,

with every undesired term in the denominator defined according to the involved technique, and evaluated in the corresponding delay-Doppler point $(\nu, \tau)$. Two examples of $\rho_{k}(\nu, \tau)$ are shown in Fig. 4 taking the values obtained from each one of the simulations performed in Fig. 2 and Fig. 3 for cGNSS-R and iGNSS-R respectively.

Finally, the SNR degradation as a function of the undesired terms is defined with respect to the thermal SNR (i.e. $\rho_{k_{\text {thermal }}}$ ). The latter is the quotient between the useful signal and the corresponding thermal noise. Then, the SNR can also be expressed in the following way:

$$
\rho_{k}(\nu, \tau)=\rho_{k_{\text {thermal }}}(\nu, \tau) \Delta_{k}(\nu, \tau),
$$

where $\Delta_{k}$ represents SNR degradation corresponding to the combined effect of cross-talk, cross-sat and RFI terms. Furthermore, SNR degradation introduced by cross-sat and RFI terms are further studied in subsequent sections. 


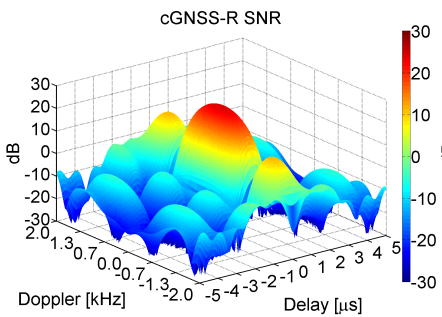

(a)

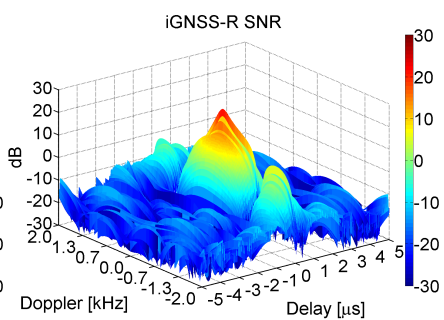

(b)
Fig. 4: Point-to-point SNR in the delay-Doppler plane obtained from (10). Each subfigure corresponds to: (a) cGNSS-R technique taking the terms depicted in Fig. 2, and (b) iGNSS-R technique according to the terms in Fig. 3.

\section{CROSS-SAT SNR DEGRADATION}

The cross-sat effect appears when one or more GNSS signals, apart from the one that is used to perform the GNSS-R measurements, are captured by the instrument antennas, and they are not completely rejected by the front-end. So that, it represents the contribution of all the cross-correlations between these satellite signals that share the same band of operation, or at least their PSD are partially overlapped. Moreover, cross-sat determines the level of intra- and interGNSS interference that is fixed by design, and it is evaluated with the SSC parameter, first used in [4]. The SSC between two signals $x$ and $y$ is defined as:

$$
\kappa_{x y}(\nu)=\int_{B_{r}} \overline{S_{x}}(f) \overline{S_{y}}(f-\nu) d f=\overline{S_{x}}(\nu) * \overline{S_{y}}(\nu),
$$

where $B_{r}$ is the bandwidth of the receiver, and $\overline{S_{x}}$ and $\overline{S_{y}}$ are the normalized PSD of $x$ and $y$ respectively. The SSC is measured in $\mathrm{dB} / \mathrm{Hz}$, and it reveals the rejection or attenuation per unit of bandwidth, suffered by other GNSS signals rather than the one under correlation.

As stated previously, GNSS codes have been designed for navigation purposes, and, considering that GNSS-R takes an advantage of the properties of these signals in an opportunistic way, it seems reasonable to study how much this overlapping affects to GNSS-R measurements, and in particular, to the SNR. Furthermore, cross-talk effect also takes place under the same conditions for the iGNSS-R technique, and its impact on the SNR may be even worse. Thus, the following evaluation will be considered valid if cross-talk effect is not present.

\section{A. SSC values}

The magnitude of the cross-sat degradation depends on the addition of the residual power of the undesired GNSS signals at the output of the front-end, $P_{s_{r, l}}$, weighted by the SSC between each the received code and the tracked one. According to the definition, the SSC depends on the Doppler of the receiver, however, the dependence can be neglected since the Doppler variations are much smaller as compared to $B_{r}$. Then, SSC is taken as constant with $\nu$, similarly to the approximation applied with the noise term. SSC values between the main GNSS codes are shown in Table I for upper
L-band (1559-1610 MHz), and in Table II for lower L-band (1164-1300 MHz). These values have been obtained using (12), and taking $B_{r}$ equal to the main lobe bandwidth as in [23].

Since cross-sat degradation is highly dependent on the scenario: number, type, position, velocity, and emitted power of satellites; signal attenuation and scattering at the reflection surface; and antennas and front-end of the receiver; an assessment of the SNR degradation induced by the cross-sat phenomenon must be performed in each particular case. For the sake of simplicity, a general case is discussed for cGNSS-R and iGNSS-R in the following subsections.

\section{B. Cross-sat in cGNSS-R}

The cross-sat term in cGNSS-R can be expressed as (see proof in Appendix C):

$$
\sum_{\substack{l \geq 0 \\ l \neq k}}^{L-1}|\chi|_{s_{r, l} c_{k}}^{2} \simeq \frac{1}{T_{c}} \sum_{\substack{l \geq 0 \\ l \neq k}}^{L-1} P_{s_{r, l}} \kappa_{s_{r, l} c_{k}}
$$

and the SNR degradation when only this term is present as:

$$
\Delta_{k_{\text {cross-sat }}} \simeq\left(1+\frac{\sum_{l \neq k}^{L-1} P_{s_{r, l}} \kappa_{s_{r, l} c_{k}}}{N_{0_{r}}}\right)^{-1} .
$$

An example of SNR degradation induced by cross-sat effect in cGNSS-R is depicted in Fig. 5. GNSS satellites from the same constellation have antenna patterns shaped to produce nearly constant power density at surface level regardless of satellite elevation. Transmitted power is also monitored and kept constant with a limited margin However, small differences are expected due to aging. For the sake of simplicity, equal received power after the front-end for all interfering satellites has been assumed, as well as the different transmitted power for different codes. Nevertheless, the received power from each satellite is not a priori known, but a minimum received power for each service is guaranteed (see Table I and II). Given this, the assumption of equal received power (and isotropic antennas) allows to calculate approximate values of the impact of cross-sat signals on the SNR. Further results about crosssat effect in GNSS-R must include particular geometry and antenna pattern case by case.

According to results depicted in Fig. 5, $\Delta_{k_{\text {cross-sat }}}$ may represent a degradation of several $\mathrm{dB}$ in case a non-directive antenna is used. However, highly directive antennas, of the order of $30 \mathrm{~dB}$, can reduce the cross-sat effect to smaller values, although the effect produced by non-used codes from the tracked satellite will still remain.

\section{Cross-sat in iGNSS-R}

The cross-sat term in iGNSS-R can be expressed as:

$$
\begin{aligned}
|\chi|_{\text {cross-sat }}^{2} \simeq & \sum_{\substack{l \geq 0 \\
l \neq k}}^{L-1}\left(\frac{1}{T_{c}} P_{s_{r, l}} \sum_{k=0}^{L-1} P_{s_{d, k}} \kappa_{s_{r, l} s_{d, k}}+\right. \\
& \left.+\frac{N_{0_{r}}}{T_{c}} P_{s_{d, l}}+P_{s_{r, l}} \frac{N_{0_{d}}}{T_{c}}\right)
\end{aligned}
$$


TABLE I: SSC values between main codes at upper GNSS band obtained from (12) in $\mathrm{dB} / \mathrm{Hz}$. SSC values depend on which is the GNSS-R receiver bandwidth $B_{r}$, the spectrum of tracked GNSS signal used in cross-correlation, the spectrum of the cross-sat signals, and the bandwidth where they are transmitted in, $B_{t}$. Infinite negative values indicate no overlapping between GNSS signal spectra. In addition, the last row shows additional information about minimum received power for each GNSS service.

\begin{tabular}{|c|c|c|c|c|c|c|c|c|c|}
\hline \multirow{2}{*}{\multicolumn{2}{|c|}{$\begin{array}{l}\text { SSC }[\mathrm{dB} / \mathrm{Hz}] \\
\text { Upper Band }\end{array}$}} & Crossed & \multirow{2}{*}{\multicolumn{4}{|c|}{$\begin{array}{c}\text { GPS } \\
30.69 \\
\end{array}$}} & \multicolumn{2}{|c|}{ Galileo } & \multirow{3}{*}{$\begin{array}{c}\text { Beidou } \\
16.00 \\
\text { B1 }\end{array}$} \\
\hline & & $B_{t}[\mathrm{MHz}]$ & & & & & \multirow{2}{*}{$\begin{array}{l}25.552 \\
\text { E1OS }\end{array}$} & \multirow{2}{*}{$\begin{array}{c}40.92 \\
\text { E1PRS }\end{array}$} & \\
\hline Tracked & $B_{r}[\mathrm{MHz}]$ & & L1CA & L1C & L1P & L1M & & & \\
\hline \multirow{4}{*}{ GPS } & 2.046 & L1CA & -61.81 & -68.28 & -70.21 & -92.41 & -68.26 & -124.50 & $-\infty$ \\
\hline & 14.322 & L1C & -68.16 & -65.46 & -70.44 & -82.94 & -65.44 & -103.74 & -106.37 \\
\hline & 20.46 & L1P & -69.90 & -70.43 & -71.25 & -79.97 & -70.41 & -101.47 & -101.48 \\
\hline & 30.69 & L1M & -87.06 & -81.86 & -79.79 & -71.55 & -81.86 & -86.72 & -82.10 \\
\hline \multirow[t]{2}{*}{ Galileo } & 14.322 & E1OS & -68.13 & -65.44 & -70.42 & -82.93 & -65.42 & -103.73 & -106.36 \\
\hline & 35.805 & E1PRS & -99.08 & -94.24 & -86.44 & -86.72 & -101.15 & -68.45 & -69.98 \\
\hline Beidou & 4.092 & B1 & -99.47 & -95.63 & -86.94 & -87.49 & -120.39 & -73.02 & -70.68 \\
\hline \multicolumn{3}{|c|}{ "Minimum Received Power [dBW] } & -161.5 & -160.0 & -164.5 & -160.0 & -157.0 & -157.0 & -157.0 \\
\hline
\end{tabular}

TABLE II: SSC between main codes at lower GNSS band. The structure is equivalent to that presented in Table I.

\begin{tabular}{|c|c|c|c|c|c|c|c|c|c|c|c|c|c|}
\hline \multirow{2}{*}{\multicolumn{2}{|c|}{$\begin{array}{l}\text { SSC }[\mathrm{dB} / \mathrm{Hz}] \\
\text { Lower Band }\end{array}$}} & Crossed & \multicolumn{4}{|c|}{ GPS } & \multicolumn{3}{|c|}{ Galileo } & \multirow{2}{*}{\multicolumn{3}{|c|}{$\begin{array}{c}\text { Beidou } \\
36.00 \\
\end{array}$}} & \multirow{3}{*}{$\begin{array}{c}\text { GLONASS } \\
20.46 \\
\text { L3OC }\end{array}$} \\
\hline & & \multirow{2}{*}{$B_{t}[\mathrm{MHz}]$} & \multicolumn{3}{|c|}{30.69} & \multirow{2}{*}{$\begin{array}{c}24.00 \\
\mathbf{L 5}\end{array}$} & \multirow{2}{*}{$\begin{array}{c}51.15 \\
\mathbf{E 5}\end{array}$} & \multicolumn{2}{|c|}{40.92} & & & & \\
\hline Tracked & $B_{r}[\mathrm{MHz}]$ & & L2C & L2P & L2M & & & E6CS & E6PRS & B2OS & B2AS & B3 & \\
\hline \multirow{4}{*}{ GPS } & 2.046 & L2C & -61.81 & -70.21 & -92.41 & $-\infty$ & $-\infty$ & $-\infty$ & $-\infty$ & $-\infty$ & $-\infty$ & $-\infty$ & $-\infty$ \\
\hline & 20.46 & L2P & -69.90 & -71.25 & -79.97 & $-\infty$ & $-\infty$ & $-\infty$ & $-\infty$ & -99.58 & -90.79 & $-\infty$ & $-\infty$ \\
\hline & 30.69 & L2M & -87.06 & -79.79 & -71.55 & $-\infty$ & -95.73 & $-\infty$ & $-\infty$ & -93.40 & -89.57 & $-\infty$ & $-\infty$ \\
\hline & 20.46 & L5 & $-\infty$ & $-\infty$ & $-\infty$ & -71.00 & -74.17 & $-\infty$ & $-\infty$ & $-\infty$ & $-\infty$ & $-\infty$ & $-\infty$ \\
\hline \multirow{3}{*}{ Galileo } & 51.15 & E5 & -110.38 & -98.14 & -95.73 & -74.17 & -74.30 & $-\infty$ & $-\infty$ & -73.12 & -74.35 & $-\infty$ & -76.18 \\
\hline & 10.23 & E6CS & $-\infty$ & $-\infty$ & $-\infty$ & $-\infty$ & $-\infty$ & -68.63 & -96.56 & $-\infty$ & $-\infty$ & -85.82 & $-\infty$ \\
\hline & 30.69 & E6PRS & $-\infty$ & $-\infty$ & $-\infty$ & $-\infty$ & $-\infty$ & -85.88 & -71.34 & $-\infty$ & $-\infty$ & -73.48 & $-\infty$ \\
\hline \multirow{3}{*}{ Beidou } & 4.092 & B2OS & $-\infty$ & $-\infty$ & $-\infty$ & $-\infty$ & -73.33 & $-\infty$ & $-\infty$ & -64.78 & -70.32 & $-\infty$ & -73.93 \\
\hline & 20.46 & B2AS & -107.43 & -95.18 & -92.84 & $-\infty$ & -74.36 & $-\infty$ & $-\infty$ & -70.11 & -71.40 & $-\infty$ & -73.36 \\
\hline & 20.46 & $\mathbf{B 3}$ & $-\infty$ & $-\infty$ & $-\infty$ & $-\infty$ & $-\infty$ & -83.39 & -73.45 & $-\infty$ & $-\infty$ & -71.40 & $-\infty$ \\
\hline GLONASS & 20.46 & L3OC & $-\infty$ & $-\infty$ & $-\infty$ & $-\infty$ & -76.18 & $-\infty$ & $-\infty$ & -73.53 & -73.35 & $-\infty$ & -70.98 \\
\hline \multicolumn{3}{|c|}{ "Minimum Received Power [dBW] } & -161.5 & -164.5 & -160.0 & -157.0 & -152.0 & -155.0 & $\begin{array}{c}-155.0 \\
\end{array}$ & -163.0 & -163.0 & -160.0 & - \\
\hline
\end{tabular}

according to Appendices $\mathrm{B}$ and $\mathrm{C}$. In this case, the corresponding cross-correlations between $n_{r}$ and $s_{d_{l}}$, and between $s_{r_{l}}$ and $n_{d}$, must be considered in addition to the combination between all non-tracked codes. The degradation $\Delta_{k_{\text {cross-sat }}}$ is defined analogously to the previous case. Besides, SSC values in Tables I and II must be recalculated using $B_{r}$ equal to the composite signal bandwidth in each case.

Figure 6 illustrates the same example of Fig. 5, but considering the iGNSS-R technique. SNR degradation levels in iGNSS-R are similar to the ones seen in cGNSS-R except in GPS composite L1 which are substantially worse as compared to L1 C/A. This is mainly given by the difference in receiver bandwidth $B_{r}$ used in each case. In cGNSS-R using GPS L1 C/A, $B_{r}=2.046 \mathrm{MHz}$, whereas $B_{r}=30.69 \mathrm{MHz}$ in iGNSS-R with GPS L1 band. Larger bandwidth implies more cross-sat power is received. In addition, when comparing the iGNSS-R cross-sat degradation in L1/E1 band with L5/E5 band, L1/E1 band is worse because it is more "crowded" than L5/E5 band. iGNSS-R L1 has slightly better performance than E1 because receiver bandwidth is also slightly smaller $(30.69 \mathrm{MHz}$ vs 35.805 MHz). The same happens when comparing L5 and
E5 (20.46 MHz vs 51.315 MHz).

\section{RFI SNR DEGRADATION}

RFI signals, other than interfering GNSS codes, can degrade or totally disrupt the performance of GNSS-R devices. The degradation of the SNR produced by RFI signals have also been studied in GNSS using the SSC, and the level of interference is highly dependent on the kind of RFI present. However, the approach followed in the state of the art is only limited to stationary RFI signals.

\section{A. Generalized SSC}

In order to evaluate the impact of any RFI (stationary or non-stationary) in GNSS-R DDMs, an extension of the SSC concept named Generalized SSC (GSSC) is introduced in this work. The GSSC between two signals $x$ and $y$ after the frontend is defined as:

$$
\gamma_{x y}(\nu, \tau)=\frac{1}{T_{c}} \int_{B_{r}} \int_{T_{c}} \overline{W_{x}}(t, f) \overline{W_{y}}(t-\tau, f-\nu) d t d f
$$




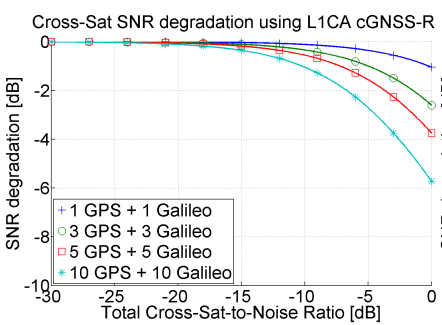

(a)

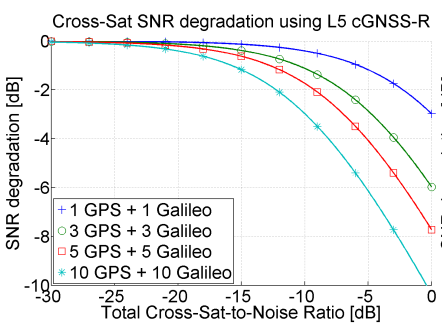

(c)

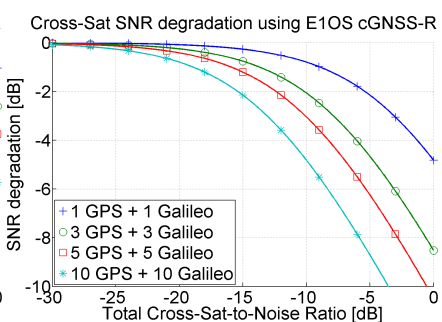

(b)

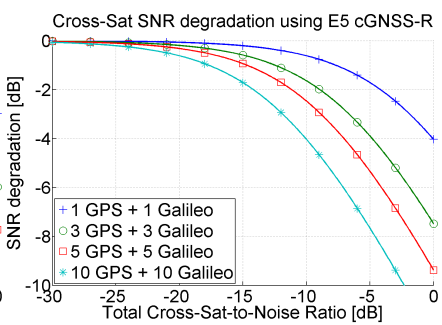

(d)

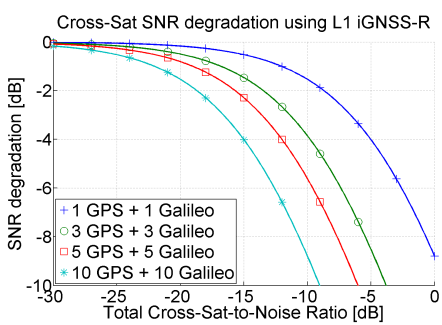

(a)

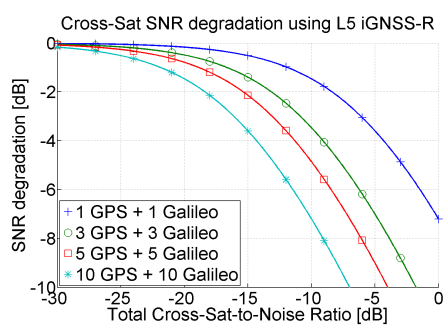

(c)

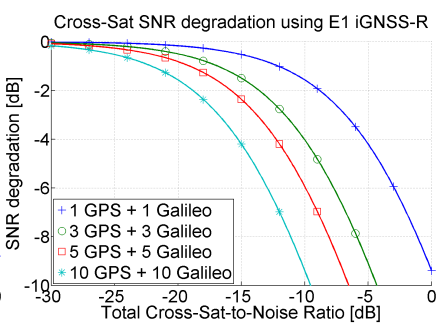

(b)
Fig. 5: Sample SNR degradation induced by cross-sat effect in cGNSS-R, and obtained from (13) and (14). The following assumptions are considered: equal received power after the front-end for all satellites, isotropic antennas, and SSC values and minimum received power stated in Tables I and II. Each subfigure corresponds to cGNSS-R using (a) GPS L1 C/A, (b) Galileo E1 OS, (c) GPS L5, and (d) Galileo E5. The total Cross-Sat-to-Noise ratio refers to the sum of power received from all interfering GPS and Galileo satellites, divided by the thermal noise power with $N_{0_{r}}=-204 \mathrm{dBW} / \mathrm{Hz}$.

where $\overline{W_{x}}$ and $\overline{W_{y}}$ are the normalized Wigner-Ville Spectrum (WVS) [24], or non-stationary spectrum, of $x$ and $y$ respectively. The WVS is used instead of the PSD defined only for stationary random processes, and its use accounts for the nonstationary nature of RFI, whose spectrum may change over the time.

\section{B. RFI in cGNSS-R}

In cGNSS-R, the RFI term can be expressed using the GSSC as follows (see proof in Appendix C):

$$
|\chi|_{i_{r} c_{k}}^{2} \simeq \frac{1}{T_{c}} P_{i_{r}} \gamma_{i_{r} c_{k}}
$$

where $P_{i_{r}}$ is the RFI power at the reflected antenna, $T_{c}$ is the coherent integration time, and $\gamma_{i_{r} c_{k}}$ is the GSSC, obtained from (16), between the RFI at the reflected antenna $i_{r}$ and the clean code replica $c_{k} . \gamma_{i_{r} c_{k}}$ has been approximated as a constant taking into account the same assumptions as for the SSC case. Moreover, the SNR degradation when this is the dominant term is:

$$
\Delta_{k_{R F I}} \simeq\left(1+\frac{P_{i_{r}} \gamma_{i_{r} c_{k}}}{N_{0_{r}}}\right)^{-1} .
$$

In order to validate this model, a commercial chirp jammer that emits a $15 \mathrm{MHz}$ frequency modulated signal centered at L1 band has been used. Figure 7 shows a comparison between the theoretical SNR degradation obtained using (18), and the one
Fig. 6: Sample SNR degradation induced by cross-sat effect in iGNSS-R, and obtained from (15) and (14). The following assumptions are considered: equal received power after the front-end for all satellites, isotropic antennas, and SSC values and minimum received power stated in Tables I and II. Each subfigure corresponds to iGNSS-R using (a) GPS composite L1 (C/A, C, P(Y), and M codes), (b) Galileo composite E1 (OS and PRS codes), (c) GPS L5, and (d) Galileo E5. The total Cross-Sat-to-Noise ratio refers to the sum of power received from all interfering GPS and Galileo satellites, divided by the interferometric noise power with $N_{0_{r}}=N_{0_{d}}=-204 \mathrm{dBW} / \mathrm{Hz}$.

measured at the laboratory using the jammer with three commercial GPS L1 C/A receivers. Theoretical SNR degradation predicted by the model shows that from $25 \mathrm{~dB}$ of Interferenceto-Noise Ratio (INR) on, SNR degradation changes has -10 $\mathrm{dB}$ per decade. Comparing this with the curves obtained from the three GPS receivers, they show a trend similar to the theoretical degradation, but their results are between 5 and 15 $\mathrm{dB}$ better, mainly because they incorporate some mitigation techniques. Furthermore, the improved performance using a custom RFI mitigation system, described in [25], also shows the same trend, but the resilience against the RFI signal has been increased $30 \mathrm{~dB}$ more. This result is twice valuable. On the one hand, the theoretical model has been validated with real measurements obtained from commercial systems. On the other hand, it demonstrates that mitigation techniques are the solution to reduce the impact of external RFI signals in GNSS$\mathrm{R}$ measurements. 


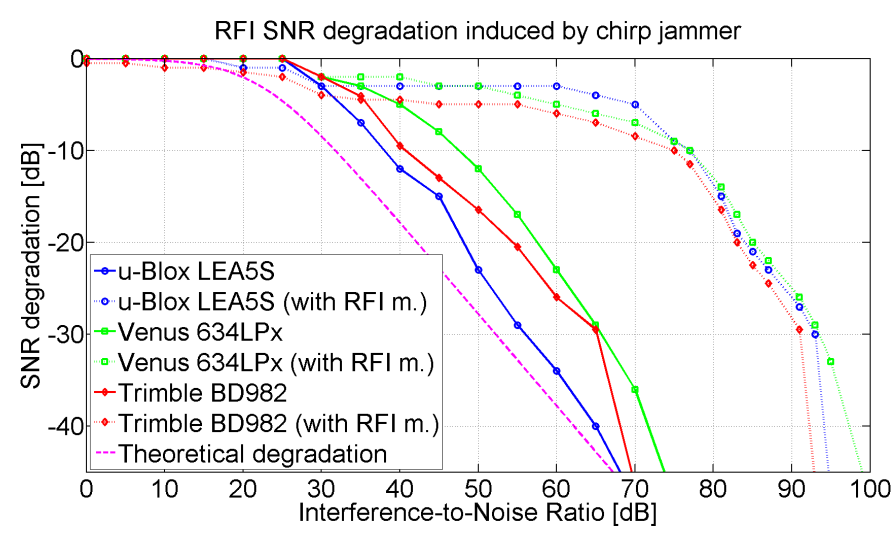

Fig. 7: Sample SNR degradation induced by RFI effect in cGNSS-R. The simulated RFI signal is a $15 \mathrm{MHz}$ sweep chirp jammer cross-correlated with GPS L1 C/A code. Theoretical result is compared to real measurements obtained from several commercial GPS receivers with and without a custom RFI mitigation system implemented at UPC-BarcelonaTech [25].

\section{RFI in iGNSS-R}

In iGNSS-R, the RFI term can be expressed as follows (see Appendices B and C):

$$
\begin{array}{r}
|\chi|_{R F I}^{2}(\nu, \tau) \simeq \frac{1}{T_{c}}\left(\sum_{l=0}^{L-1} P_{s_{r, l}} P_{i_{d}} \gamma_{s_{r, l} i_{d}}\right. \\
+\sum_{l=0}^{L-1} P_{i_{r}} P_{s_{d, l}} \gamma_{i_{r} s_{d, l}} \\
+P_{i_{r}} P_{i_{d}} \gamma_{i_{r} i_{d}}(\nu, \tau)+P_{i_{r}} \frac{N_{0_{d}}}{T_{c}} \gamma_{i_{r} n_{d}} \\
\left.+\frac{N_{0_{r}}}{T_{c}} P_{i_{d}} \gamma_{n_{r} i_{d}}\right),
\end{array}
$$

and the SNR degradation can be defined in an analogous way to the cGNSS-R case. In iGNSS-R, four sub-terms contribute constantly to the SNR degradation whereas there is only one that depends on $\nu$ and $\tau . \gamma_{i_{r} i_{d}}(\nu, \tau)$ corresponds to the crosscorrelation between RFI signals captured by both direct and reflected antennas, and it can reach magnitudes much higher than the useful signal, even if high directive antennas are used.

In order to illustrate the effects of RFI signals in iGNSSR, GSSC values in (19) are depicted in Fig. 8 for the case of a DME RFI signal interfering GPS L5 and Galileo E5 iGNSS-R measurements. It can be appreciated that the GSSC terms $\gamma_{s_{r, l} i_{d}}, \gamma_{i_{r} s_{d, l}}, \gamma_{i_{r} n_{d}}$, and $\gamma_{n_{r} i_{d}}$ are of the order of the SSC values obtained in Section III for cross-sat terms, but they may depend on the DME frequency channel. However, if the received RFI power represented by $P_{i_{d}}$ and $P_{i_{r}}$ is much higher than the received noise and satellite signal power, the effect of RFI SNR degradation will be worse than in the cross-sat case. Moreover, the GSSC corresponding to a crosscorrelation between direct and reflected DME signals, $\gamma_{i_{r} i_{d}}$, is concentrated in few delay lags rather that spread over the whole delay-Doppler space. This fact implies that the SNR degradation around this peak will be much higher as compared to other terms. Further information about the impact of DME signals on iGNSS-R measurements can be found in [26].

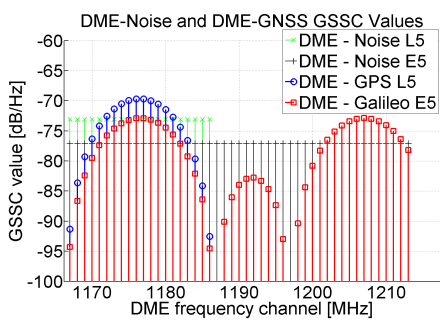

(a)

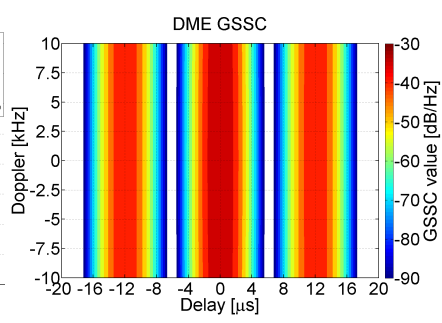

(b)
Fig. 8: Sample GSSC values obtained using (16). This example considers an iGNSS-R instrument interfered with a DME RFI signal. DME spectrum is overlapped with GPS L5 and Galileo E5 spectra. Each subfigure corresponds to (a) the GSSC values for crossed combinations DME to Noise, and DME to GNSS signals, and (b) GSSC values for DME signals present at both direct and reflected antennas.

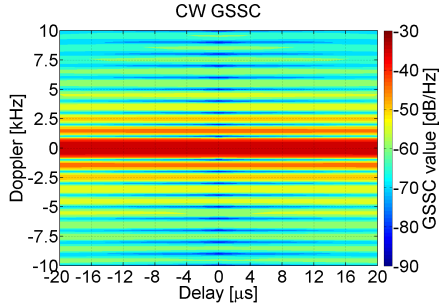

(a)

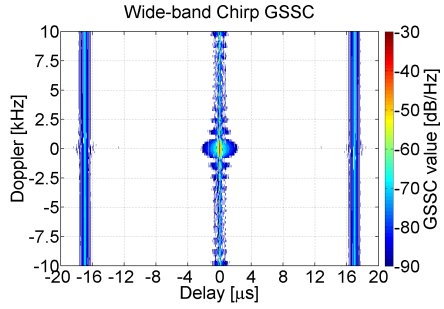

(c)

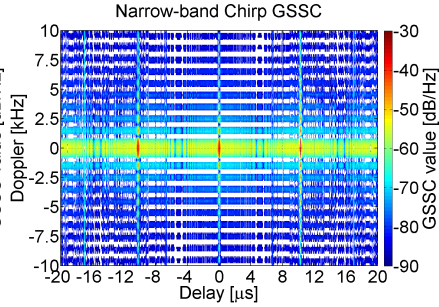

(b)

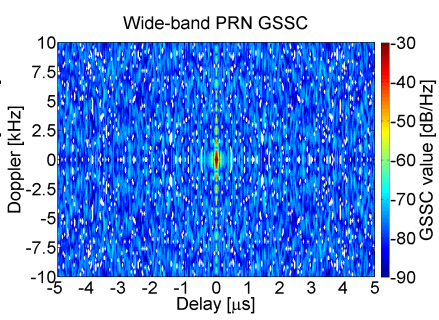

(d)
Fig. 9: Sample GSSC values obtained using (16) considering GPS L1 iGNSS-R with $B_{r}=30.69 \mathrm{MHz}$ under different RFI signals. Each subfigure corresponds to: (a) a $\mathrm{CW}$ signal, (b) a narrow-band chirp with $B_{\text {chirp }}=15 \mathrm{MHz}$ and $10.24 \mu \mathrm{s}$ repetition period, (c) a wide-band chirp with $B_{\text {chirp }}=60 \mathrm{MHz}$ and $81.92 \mu \mathrm{s}$ repetition period, and (d) a Pseudo-Random Noise (PRN) signal with $25 \mathrm{MHz}$ of bandwidth. Results are shown for GPS L1 iGNSS-R with $B_{r}=30.69 \mathrm{MHz}$.

As stated above, the GSSC between direct and reflected signals in iGNSS-R may increase the SNR degradation considerably. For the sake of completeness the GSSC values corresponding to four typical RFI signal shapes [27] are shown in Fig. 9. The chosen RFI signals are a sinusoidal or Continuous Wave (CW) signal, a narrow-band chirp with $B_{\text {chirp }}=15 \mathrm{MHz}$ and $10.24 \mu s$ repetition period, a wide-band chirp with $B_{\text {chirp }}=60 \mathrm{MHz}$ and $81.92 \mu s$ repetition period, and a Pseudo-Random Noise (PRN) signal with $25 \mathrm{MHz}$ of bandwidth. Results are shown for GPS L1 iGNSS-R with $B_{r}$ $=30.69 \mathrm{MHz}$. 
Figure 9 depicts how RFI signals whose power is more concentrated in frequency, such as $\mathrm{CW}$ and narrow-band chirp, are more spread in the delay domain after the correlation process. On the contrary, wide-band chirp and PRN RFI signals are more concentrated in delay domain. This behavior is explained taking into account that delay and frequency are complementary domains [28]. Moreover, the behavior in the Doppler domain will depend on the RFI signal shape itself.

$\mathrm{CW}$, and in general narrow-band signals, are in principle more troublesome for GNSS-R measurements since they are more spread in the delay-Doppler space. Conversely, wideband signals will only degrade the SNR at particular delay bins, but they can be fatal if the tracked signal DDM information is also in these bins. Therefore, the probability of SNR degradation will be higher for narrow-band signals than for wide-band signals. However, mitigation techniques are more effective for narrow-band than for than wide-ban signals.

\section{Conclusions}

RFI signals can overwhelm the performance of GNSS-R instruments as it was demonstrated in in the past (e.g. [2]). In this paper, a model to evaluate the degradation of the SNR in GNSS-R measurements has been proposed for both cGNSS$\mathrm{R}$ and iGNSS-R techniques, and it has been evaluated with a general case for each technique.

Regarding cGNSS-R, the degradation of the thermal SNR can be produced by either cross-sat effect, or external RFI effect, or both. Moreover, they have a constant degradation over the delay-Doppler space. The cross-sat effect may represent degradation of more than $10 \mathrm{~dB}$ considering several satellites with all existing and forthcoming GNSS signals. Nevertheless, the cross-sat effect can be neglected when using highly directive antennas. In particular, antenna arrays with beam-steering capabilities are able to track a specific satellite, while they attenuate signals from the rest of them. On the other hand, the external RFI effect may represent a corruption of the measurements since it is known that they can disrupt the performance of GNSS receivers. In this case, mitigation techniques must be used in order to reduce the error produced by the RFI effect [27].

The iGNSS-R technique has been proven to be much more sensitive to RFI than cGNSS-R. Moreover, in this case, a new phenomenon is introduced: the cross-talk effect that may represent a total corruption of the measurements. Crosstalk together with cross-sat effect may also be attenuated with directive antennas. Finally, external RFI effect can have a catastrophic effect in the measurements, even worse as compared to cGNSS-R, if the interference is captured by both antennas, reflected and direct. In this case, the use of highly directive antennas can help to decorrelate the signals captured by them. However, the RFI effect will still remain because of the multiple residual cross-correlations between RFI signals and thermal noise captured by both antennas. Therefore, mitigation techniques will be essential to improve the performance of iGNSS-R instruments under RFI conditions.

A specific mention must be given to former GLONASS which uses spread-spectrum signals with a single code combined with Frequency Division Multiple Access (FDMA). Due to the use of different frequencies for different satellites, crosstalk and cross-sat effects will disappear in both cGNSS-R and iGNSS-R techniques, even if non-directive antennas are used. Nevertheless, external RFI signals are still troublesome. With the conventional approach, if the RFI power is well concentrated in frequency (for instance a CW RFI signal), the SNR degradation could be much higher for some GLONASS satellites (those where the RFI signal overlaps their respective spectra), as compared to GPS or Galileo satellites. The opposite would occur if the RFI signal is wide-band. Moreover, in the interferometric approach, SNR degradation would be of similar order since the cross-correlated term between direct and reflected signal does not depend on the used GNSS.

\section{APPENDIX A}

\section{DERIVATION OF DDM DECOMPOSITION IN CGNSS-R}

The CWAF has been defined as the operation needed to calculate the measured DDM in GNSS-R, and, for two generic signals $x$ and $y$, it is computed as:

$$
|\chi|_{x y}^{2}(\nu, \tau)=\mathrm{E}\left\{\left|\chi_{x y}(\nu, \tau)\right|^{2}\right\} .
$$

In the state of the art, the Sussman's formula was introduced in [20] as a general proof of several properties of ambiguity functions such as the Siebert's theorem [29] and the Stutt's invariant relation [30], and states that if a Cross-Ambiguity Function (CAF) is defined as:

$$
\chi_{x y}(\nu, \tau)=\int x(t) y^{*}(t-\tau) e^{-j 2 \pi \nu t} d t,
$$

the following identity is fulfilled:

$$
\begin{aligned}
& \chi_{x y}(\nu, \tau) \chi_{w z}^{*}(\nu, \tau) \\
& \quad=\iint \chi_{x w}\left(\nu^{\prime}, \tau^{\prime}\right) \chi_{y z}^{*}\left(\nu^{\prime}, \tau^{\prime}\right) e^{j 2 \pi\left(\nu^{\prime} \tau-\nu \tau^{\prime}\right)} d \nu^{\prime} d \tau^{\prime},
\end{aligned}
$$

so that, the modulus squared of any CAF can be expressed as:

$$
\begin{aligned}
& \left|\chi_{x y}(\nu, \tau)\right|^{2} \\
& \quad=\chi_{x y}(\nu, \tau) \chi_{x y}^{*}(\nu, \tau) \\
& \quad=\iint \chi_{x x}\left(\nu^{\prime}, \tau^{\prime}\right) \chi_{y y}^{*}\left(\nu^{\prime}, \tau^{\prime}\right) e^{j 2 \pi\left(\nu^{\prime} \tau-\nu \tau^{\prime}\right)} d \nu^{\prime} d \tau^{\prime} .
\end{aligned}
$$

This expression was derived for infinite coherent integration time, however, this is always finite. In order to obtain an expression equivalent to (23) for finite $T_{c}$, the equation defined for the delay-Doppler correlator in (3) is modulus squared as follows:

$$
\begin{aligned}
\left|\chi_{x y}(\nu, \tau)\right|^{2} & =\chi_{x y}(\nu, \tau) \chi_{x y}^{*}(\nu, \tau) \\
& =\left[\frac{1}{T_{c}} \int_{T_{c}} x\left(t_{1}\right) y^{*}\left(t_{1}-\tau\right) e^{-j 2 \pi \nu t_{1}} d t_{1}\right] \\
& {\left[\frac{1}{T_{c}} \int_{T_{c}} x\left(t_{2}\right) y^{*}\left(t_{2}-\tau\right) e^{-j 2 \pi \nu t_{2}} d t_{2}\right]^{*} } \\
& =\iint \frac{1}{T_{c}} \Pi\left(\frac{t_{1}}{T_{c}}\right) \frac{1}{T_{c}} \Pi\left(\frac{t_{2}}{T_{c}}\right) x\left(t_{1}\right) y^{*}\left(t_{1}-\tau\right) \\
& x^{*}\left(t_{2}\right) y\left(t_{2}-\tau\right) e^{-j 2 \pi \nu\left(t_{1}-t_{2}\right)} d t_{1} d t_{2} .
\end{aligned}
$$


Applying the change of variables $t_{1}=t$ and $t_{2}=t-\tau^{\prime}$ with $d t_{1} d t_{2}=\left|J\left(t, \tau^{\prime}\right)\right| d t d \tau^{\prime}=d t d \tau^{\prime}$ being $J$ the Jacobian matrix of the transformation, (24) yields:

$$
\begin{aligned}
& \left|\chi_{x y}(\nu, \tau)\right|^{2} \\
& \quad=\iint \frac{1}{T_{c}} \Pi\left(\frac{t}{T_{c}}\right) x(t) x^{*}\left(t-\tau^{\prime}\right) \\
& \quad \frac{1}{T_{c}} \Pi\left(\frac{t-\tau^{\prime}}{T_{c}}\right) y^{*}(t-\tau) y\left(t-\tau-\tau^{\prime}\right) e^{-j 2 \pi \nu \tau^{\prime}} d t d \tau^{\prime},
\end{aligned}
$$

Then, using the inverse of the identities:

$$
\begin{aligned}
\tilde{\chi}_{x x}\left(\nu^{\prime}, \tau^{\prime}\right) & =\int \frac{1}{T_{c}} \Pi\left(\frac{t}{T_{c}}\right) x(t) x^{*}\left(t-\tau^{\prime}\right) e^{-j 2 \pi \nu^{\prime} t} d t \\
& =\operatorname{sinc}\left(T_{c} \nu^{\prime}\right) \underset{\nu^{\prime}}{*} \chi_{x x}\left(\nu^{\prime}, \tau^{\prime}\right),
\end{aligned}
$$

and

$$
\begin{aligned}
\tilde{\chi}_{y y}\left(\nu^{\prime \prime}, \tau^{\prime}\right) & =\int \frac{1}{T_{c}} \Pi\left(\frac{t-\tau^{\prime}}{T_{c}}\right) y^{*}(t-\tau) y\left(t-\tau-\tau^{\prime}\right) \\
& e^{-j 2 \pi \nu^{\prime \prime}(t-\tau)} d t \\
& =\left[\operatorname{sinc}\left(T_{c} \nu^{\prime \prime}\right) e^{-j 2 \pi \nu^{\prime \prime}\left(\tau^{\prime}-\tau\right)}\right]_{\nu^{\prime \prime}}^{*} \chi_{y y}\left(\nu^{\prime \prime}, \tau^{\prime}\right),
\end{aligned}
$$

(25) can be expressed as:

$$
\begin{aligned}
& \left|\chi_{x y}(\nu, \tau)\right|^{2} \\
& =\iint\left[\int \tilde{\chi}_{x x}\left(\nu^{\prime}, \tau^{\prime}\right) e^{j 2 \pi \nu^{\prime} t} d \nu^{\prime}\right] \\
& {\left[\int \tilde{\chi}_{y y}\left(\nu^{\prime \prime}, \tau^{\prime}\right) e^{j 2 \pi \nu^{\prime \prime}(t-\tau)} d \nu^{\prime \prime}\right]^{*} e^{-j 2 \pi \nu \tau^{\prime}} d t d \tau^{\prime}} \\
& =\iint \tilde{\chi}_{x x}\left(\nu^{\prime}, \tau^{\prime}\right) \int \tilde{\chi}_{y y}^{*}\left(\nu^{\prime \prime}, \tau^{\prime}\right)\left[\int e^{-j 2 \pi\left(\nu^{\prime \prime}-\nu^{\prime}\right) t} d t\right] \\
& e^{j 2 \pi \nu^{\prime \prime} \tau} e^{-j 2 \pi \nu \tau^{\prime}} d \nu^{\prime \prime} d \nu^{\prime} d \tau^{\prime} \\
& =\iint \tilde{\chi}_{x x}\left(\nu^{\prime}, \tau^{\prime}\right) \int \tilde{\chi}_{y y}^{*}\left(\nu^{\prime \prime}, \tau^{\prime}\right) \delta\left(\nu^{\prime \prime}-\nu^{\prime}\right) \\
& e^{j 2 \pi\left(\nu^{\prime \prime} \tau-\nu \tau^{\prime}\right)} d \nu^{\prime \prime} d \nu^{\prime} d \tau^{\prime} \\
& =\iint \tilde{\chi}_{x x}\left(\nu^{\prime}, \tau^{\prime}\right) \tilde{\chi}_{y y}^{*}\left(\nu^{\prime}, \tau^{\prime}\right) e^{j 2 \pi\left(\nu^{\prime} \tau-\nu \tau^{\prime}\right)} d \nu^{\prime} d \tau^{\prime},
\end{aligned}
$$

where $\tilde{\chi}_{x x}$ and $\tilde{\chi}_{y y}$ are windowed and delayed versions of $\chi_{x x}$ and $\chi_{y y}$ respectively. Indeed, the last ones correspond to the auto-correlation functions of the input signals using correlator defined in (3) as was done in [18]. Therefore, if $x$ and $y$ are already taken windowed by $T_{c}$ and delayed by $\tau, \widetilde{\chi}_{x x}=\chi_{x x}$, $\tilde{\chi}_{y y}=\chi_{y y}$, and (27) is equivalent to (23). Henceforth, this last statement will be assumed.
Eventually, substituting (27) (or equivalent (23)) in (20), and considering statistical independence between $x$ and $y$, the CWAF yields:

$|\chi|_{x y}^{2}(\nu, \tau)$

$$
\begin{aligned}
& =\mathrm{E}\left\{\left|\chi_{x y}(\nu, \tau)\right|^{2}\right\} \\
& =\mathrm{E}\left\{\iint \chi_{x x}\left(\nu^{\prime}, \tau^{\prime}\right) \chi_{y y}^{*}\left(\nu^{\prime}, \tau^{\prime}\right) e^{j 2 \pi\left(\nu^{\prime} \tau-\nu \tau^{\prime}\right)} d \nu^{\prime} d \tau^{\prime}\right\}
\end{aligned}
$$

$$
\begin{aligned}
& =\iint \mathrm{E}\left\{\chi_{x x}\left(\nu^{\prime}, \tau^{\prime}\right)\right\} \mathrm{E}\left\{\chi_{y y}^{*}\left(\nu^{\prime}, \tau^{\prime}\right)\right\} e^{j 2 \pi\left(\nu^{\prime} \tau-\nu \tau^{\prime}\right)} d \nu^{\prime} d \tau^{\prime} \\
& =\iint A_{x}\left(\nu^{\prime}, \tau^{\prime}\right) A_{y}^{*}\left(\nu^{\prime}, \tau^{\prime}\right) e^{j 2 \pi\left(\nu^{\prime} \tau-\nu \tau^{\prime}\right)} d \nu^{\prime} d \tau^{\prime},
\end{aligned}
$$

where $A_{x}$ and $A_{y}$ are the Expected Ambiguity Function (EAF) of signals $x$ and $y$ respectively. This expression will be used to prove the DDM decomposition for cGNSS-R stated in (6).

Therefore, using (28), the CWAF in (4) can be expressed as a function of $A_{y_{r}}$ and $A_{c_{k}}$. $A_{c_{k}}$ is the EAF of the tracked code, whereas $A_{y_{r}}$ is the EAF of the received signal after the front-end obtained from:

$$
A_{y_{r}}(\nu, \tau)=\frac{1}{T_{c}} \int_{T_{c}} \mathrm{E}\left\{y_{r}(t) y_{r}^{*}(t-\tau)\right\} e^{-j 2 \pi \nu t} d t .
$$

$\mathrm{E}\left\{y_{r}(t) y_{r}^{*}(t-\tau)\right\}$ can be expanded using the terms of $y_{r}$ in 
(2) as follows:

$$
\begin{aligned}
\mathrm{E}\left\{y_{r}(t) y_{r}^{*}(t-\tau)\right\}= & \mathrm{E}\left\{\left[\sum_{l=0}^{L-1} s_{r_{l}}(t)+i_{r}(t)+n_{r}(t)\right]\right. \\
& {\left.\left[\sum_{l^{\prime}=0}^{L-1} s_{r_{l^{\prime}}}^{*}(t-\tau)+i_{r}^{*}(t-\tau)+n_{r}^{*}(t-\tau)\right]\right\} } \\
= & \sum_{l=0}^{L-1} \mathrm{E}\left\{s_{r_{l}}(t) s_{r_{l}}^{*}(t-\tau)\right\} \\
& +\sum_{l \geq 0}^{L-1} \sum_{l^{\prime}=0}^{L-1} \mathrm{E}\left\{s_{r_{l}}(t) s_{r_{l^{\prime}}}^{*}(t-\tau)\right\} \\
& +\sum_{l=0}^{L-1} \mathrm{E}\left\{s_{r_{l}}(t) i_{r}^{*}(t-\tau)\right\} \\
& +\sum_{l=0}^{L-1} \mathrm{E}\left\{s_{r_{l}}(t) n_{r}^{*}(t-\tau)\right\} \\
& +\sum_{l^{\prime}=0}^{L-1} \mathrm{E}\left\{i_{r}(t) s_{r_{l^{\prime}}}^{*}(t-\tau)\right\} \\
& +\mathrm{E}\left\{i_{r}(t) i_{r}^{*}(t-\tau)\right\} \\
& +\mathrm{E}\left\{i_{r}(t) n_{r}^{*}(t-\tau)\right\} \\
& +\sum_{l^{\prime}=0}^{L-1} \mathrm{E}\left\{n_{r}(t) s_{r_{l^{\prime}}}^{*}(t-\tau)\right\} \\
& +\mathrm{E}\left\{n_{r}(t) i_{r}^{*}(t-\tau)\right\} \\
& +\mathrm{E}\left\{n_{r}(t) n_{r}^{*}(t-\tau)\right\} \\
= & \sum_{l=0}^{L-1} \mathrm{E}\left\{s_{r_{l}}(t) s_{r_{l}}^{*}(t-\tau)\right\} \\
& +\mathrm{E}\left\{i_{r}(t) i_{r}^{*}(t-\tau)\right\} \\
& +\mathrm{E}\left\{n_{r}(t) n_{r}^{*}(t-\tau)\right\} \\
& \\
& \\
&
\end{aligned}
$$

assuming that $s_{r}, i_{r}$ and $n_{r}$ are statistically independent between them, that $\mathrm{E}\left\{s_{r_{l}}(t) s_{r_{l^{\prime}}}^{*}(t-\tau)\right\}=0$ because of code orthogonality, and that $\mathrm{E}\left\{i_{r}(t)\right\}=\mathrm{E}\left\{n_{r}(t)\right\}=0$, without loss of generality. Then, (29) can be expressed as:

$$
A_{y_{r}}(\nu, \tau)=\sum_{l=0}^{L-1} A_{s_{r, l}}(\nu, \tau)+A_{i_{r}}(\nu, \tau)+A_{n_{r}}(\nu, \tau),
$$

and, eventually, the DDM decomposition stated in (6) is obtained by substituting (31) in (28) yielding:

$$
\begin{aligned}
|\chi|_{y_{r} c_{k}}^{2}(\nu, \tau)= & \sum_{l=0}^{L-1}|\chi|_{s_{r, l} c_{k}}^{2}(\nu, \tau) \\
& +|\chi|_{i_{r} c_{k}}^{2}(\nu, \tau)+|\chi|_{n_{r} c_{k}}^{2}(\nu, \tau) \\
= & |\chi|_{s_{r, k} c_{k}}^{2}(\nu, \tau)+\sum_{\substack{l>0 \\
l \neq k}}^{L-1}|\chi|_{s_{r, l} c_{k}}^{2} \\
& +|\chi|_{i_{r} c_{k}}^{2}+|\chi|_{n_{r} c_{k}}^{2},
\end{aligned}
$$

where the dependence with $\nu$ and $\tau$ in the last terms has been omitted since it does not apply. This result may be derived intuitively taking into account the statistical independence between the additive terms of the input signal.

\section{APPENDIX B}

DERIVATION OF DDM DECOMPOSITION IN IGNSS-R

The derivation of the DDM in the case of iGNSS-R can be obtained from expressions in Appendix A. First, using (28), the CWAF can be expressed as:

$$
\begin{aligned}
& |\chi|_{y_{r} y_{d}}^{2}(\nu, \tau) \\
& =\mathrm{E}\left\{\left|\chi_{y_{r} y_{d}}(\nu, \tau)\right|^{2}\right\} \\
& =\iint A_{y_{r}}\left(\nu^{\prime}, \tau^{\prime}\right) A_{y_{d}}^{*}\left(\nu^{\prime}, \tau^{\prime}\right) e^{j 2 \pi\left(\nu^{\prime} \tau-\nu \tau^{\prime}\right)} d \nu^{\prime} d \tau^{\prime},
\end{aligned}
$$

since $y_{r}$ and $y_{d}$ are assumed statistically independent. Besides, the EAF of $y_{d}$ can be written as:

$$
A_{y_{d}}(\nu, \tau)=\sum_{l^{\prime}=0}^{L-1} A_{s_{d, l^{\prime}}}(\nu, \tau)+A_{i_{d}}(\nu, \tau)+A_{n_{d}}(\nu, \tau),
$$

considering the same assumptions and derivations taken in (29), (30), and (31), but for the direct received signals in (5). Eventually, substituting (31) and (34) in (33), the interfero- 
metric CWAF yields:

$|\chi|_{y_{r} y_{d}}^{2}(\nu, \tau)$

$=\iint\left[\sum_{l=0}^{L-1} A_{s_{r, l}}\left(\nu^{\prime}, \tau^{\prime}\right)+A_{i_{r}}\left(\nu^{\prime}, \tau^{\prime}\right)+A_{n_{r}}\left(\nu^{\prime}, \tau^{\prime}\right)\right]$

$\left[\sum_{l^{\prime}=0}^{L-1} A_{s_{d, l^{\prime}}}^{*}\left(\nu^{\prime}, \tau^{\prime}\right)+A_{i_{d}}^{*}\left(\nu^{\prime}, \tau^{\prime}\right)+A_{n_{d}}^{*}\left(\nu^{\prime}, \tau^{\prime}\right)\right] e^{j 2 \pi\left(\nu^{\prime} \tau-\nu \tau^{\prime}\right)} d \nu^{\prime} d \tau^{\prime}$

$=\iint\left[\sum_{l=0}^{L-1} \sum_{l^{\prime}=0}^{L-1} A_{s_{r, l}}\left(\nu^{\prime}, \tau^{\prime}\right) A_{s_{d, l^{\prime}}}^{*}\left(\nu^{\prime}, \tau^{\prime}\right)\right.$

$+\sum_{l=0}^{L-1} A_{s_{r, l}}\left(\nu^{\prime}, \tau^{\prime}\right) A_{i_{d}}^{*}\left(\nu^{\prime}, \tau^{\prime}\right)$

$+\sum_{l=0}^{L-1} A_{s_{r, l}}\left(\nu^{\prime}, \tau^{\prime}\right) A_{n_{d}}^{*}\left(\nu^{\prime}, \tau^{\prime}\right)$

$+A_{i_{r}}\left(\nu^{\prime}, \tau^{\prime}\right) \sum_{l^{\prime}=0}^{L-1} A_{s_{d, l^{\prime}}}^{*}\left(\nu^{\prime}, \tau^{\prime}\right)$

$+A_{i_{r}}\left(\nu^{\prime}, \tau^{\prime}\right) A_{i_{d}}^{*}\left(\nu^{\prime}, \tau^{\prime}\right)+A_{i_{r}}\left(\nu^{\prime}, \tau^{\prime}\right) A_{n_{d}}^{*}\left(\nu^{\prime}, \tau^{\prime}\right)$

$+A_{n_{r}}\left(\nu^{\prime}, \tau^{\prime}\right) \sum_{l^{\prime}=0}^{L-1} A_{s_{d, l^{\prime}}}^{*}\left(\nu^{\prime}, \tau^{\prime}\right)+A_{n_{r}}\left(\nu^{\prime}, \tau^{\prime}\right) A_{i_{d}}^{*}\left(\nu^{\prime}, \tau^{\prime}\right)$

$\left.+A_{n_{r}}\left(\nu^{\prime}, \tau^{\prime}\right) A_{n_{d}}^{*}\left(\nu^{\prime}, \tau^{\prime}\right)\right] e^{j 2 \pi\left(\nu^{\prime} \tau-\nu \tau^{\prime}\right)} d \nu^{\prime} d \tau^{\prime}$

$=\sum_{l=0}^{L-1} \sum_{l^{\prime}=0}^{L-1}|\chi|_{s_{r, l} s_{d, l^{\prime}}}^{2}(\nu, \tau)+\sum_{l=0}^{L-1}|\chi|_{s_{r, l} i_{d}}^{2}(\nu, \tau)$

$+\sum_{l=0}^{L-1}|\chi|_{s_{r, l} n_{d}}^{2}(\nu, \tau)+\sum_{l=0}^{L-1}|\chi|_{i_{r} s_{d, l}}^{2}(\nu, \tau)$

$+|\chi|_{i_{r} i_{d}}^{2}(\nu, \tau)+|\chi|_{i_{r} n_{d}}^{2}(\nu, \tau)+\sum_{l=0}^{L-1}|\chi|_{n_{r} s_{d, l}}^{2}(\nu, \tau)$

$+|\chi|_{n_{r} i_{d}}^{2}(\nu, \tau)+|\chi|_{n_{r} n_{d}}^{2}(\nu, \tau)$.

The last formula can be simplified in order to obtain the following expression for DDM decomposition for the $k$ th composite satellite signal using iGNSS-R:

$$
\begin{aligned}
|\chi|_{y_{r} y_{d}}^{2}(\nu, \tau)= & |\chi|_{s_{r, k} s_{d, k}}^{2}(\nu, \tau)+|\chi|_{\text {cross-talk }}^{2}(\nu, \tau) \\
& +|\chi|_{\text {cross-sat }}^{2}(\nu, \tau) \\
& +|\chi|_{\text {RFI }}^{2}(\nu, \tau)+|\chi|_{\text {noise }}^{2}(\nu, \tau),
\end{aligned}
$$

where the last four terms are defined as follows:

$$
\begin{aligned}
|\chi|_{\text {cross-talk }}^{2}(\nu, \tau)=\sum_{\substack{l \geq 0 \\
l \neq k}}^{L-1}|\chi|_{s_{r, l} s_{d, l}}^{2}(\nu, \tau), \\
|\chi|_{\text {cross-sat }}^{2}(\nu, \tau)=\sum_{\substack{l \geq 0 \\
l \neq l^{\prime}}}^{L-1} \sum_{l^{\prime}=0}^{L-1}|\chi|_{s_{r, l} s_{d, l^{\prime}}}^{2}(\nu, \tau) \\
+\sum_{\substack{l \geq 0 \\
l \neq k}}^{L-1}|\chi|_{s_{r, l} n_{d}}^{2}(\nu, \tau)+\sum_{\substack{l \geq 0 \\
l \neq k}}^{L-1}|\chi|_{n_{r} s_{d, l}}^{2}(\nu, \tau),
\end{aligned}
$$

$$
\begin{aligned}
|\chi|_{\mathrm{RFI}}^{2}(\nu, \tau)= & \sum_{l=0}^{L-1}|\chi|_{s_{r, l} i_{d}}^{2}(\nu, \tau)+\sum_{l=0}^{L-1}|\chi|_{i_{r} s_{d, l}}^{2}(\nu, \tau) \\
& +|\chi|_{i_{r} i_{d}}^{2}(\nu, \tau)+|\chi|_{i_{r} n_{d}}^{2}(\nu, \tau)+|\chi|_{n_{r} i_{d}}^{2}(\nu, \tau),
\end{aligned}
$$

and

$$
|\chi|_{\text {noise }}^{2}(\nu, \tau)=|\chi|_{s_{r, k} n_{d}}^{2}(\nu, \tau)+|\chi|_{n_{r} s_{d, k}}^{2}(\nu, \tau)+|\chi|_{n_{r} n_{d}}^{2}(\nu, \tau) \text {. }
$$

Eventually, (8) has been obtained from the combination of (36a) and (36e), and omitting the dependence with $\nu$ and $\tau$ in the terms that it does not apply.

\section{APPENDIX C}

\section{DERIVATION OF THE GSSC AND REDUCTION TO SSC}

A CWAF, and particularly the result obtained in (27), can be expressed in an alternative way as a function of the WVS of each one of the involved signals, which are the Fourier transform of the their respective EAF, which are related as follows [28]:

$$
A_{x}(\nu, \tau)=\frac{1}{T_{c}} \iint_{T_{c}} W_{x}(t, f) e^{-j 2 \pi(\nu t-f \tau)} d t d f
$$

with the WVS defined as:

$$
W_{x}(t, f)=\int \mathrm{E}\left\{x(t) x^{*}(t-\tau)\right\} e^{-j 2 \pi f \tau} d \tau,
$$

taking into account the time window $T_{c}$ as in Appendix A. Then, substituting (37) into (28), $|\chi|_{x y}^{2}$ can also be written as:

$|\chi|_{x y}^{2}(\nu, \tau)$

$=\iint\left[\frac{1}{T_{c}} \iint_{T_{c}} W_{x}(t, f) e^{-j 2 \pi\left(\nu^{\prime} t-f \tau^{\prime}\right)} d t d f\right]$

$\left[\frac{1}{T_{c}} \iint_{T_{c}} W_{y}\left(t^{\prime}, f^{\prime}\right) e^{-j 2 \pi\left(\nu^{\prime} t^{\prime}-f^{\prime} \tau^{\prime}\right)} d t^{\prime} d f^{\prime}\right]^{*} e^{j 2 \pi\left(\nu^{\prime} \tau-\nu \tau^{\prime}\right)} d \nu^{\prime} d \tau^{\prime}$

$=\frac{1}{T_{c}^{2}} \iint_{T_{c}} W_{x}(t, f) \iint_{T_{c}} W_{y}\left(t^{\prime}, f^{\prime}\right)\left[\int e^{j 2 \pi \nu^{\prime}\left(t^{\prime}-t+\tau\right)} d \nu^{\prime}\right]$

$\left[\int e^{-j 2 \pi\left(f^{\prime}-f+\nu\right) \tau^{\prime}} d \tau^{\prime}\right] d t^{\prime} d f^{\prime} d t d f$

$=\frac{1}{T_{c}^{2}} \iint_{T_{c}} W_{x}(t, f) \iint_{T_{c}} W_{y}\left(t^{\prime}, f^{\prime}\right) \delta\left(t^{\prime}-t+\tau\right)$

$\delta\left(f^{\prime}-f+\nu\right) d t^{\prime} d f^{\prime} d t d f$

$=\frac{1}{T_{c}^{2}} \iint_{T_{c}} W_{x}(t, f) W_{y}(t-\tau, f-\nu) d t d f$

$=\frac{1}{T_{c}^{2}} W_{x}(\tau, \nu) * * W_{y}(\tau, \nu)$,

where the reality and commutative properties of the WVS have been applied [28]. This result resembles the definition of the SSC in [31], but in this case, it involves the convolution of nonstationary spectrum functions. For this reason, a more general figure of merit is defined in this paper, which is the GSSC. The GSSC between two generic signals $x$ and $y$ is defined as:

$$
\gamma_{x y}(\nu, \tau)=\frac{1}{T_{c}} \overline{W_{x}}(\tau, \nu) * * \overline{W_{y}}(\tau, \nu)
$$


where $W_{x}$ and $W_{y}$ have been normalized dividing by their respective power $P_{x}$ and $P_{y}$, in analogy to the classical SSC. Therefore, using (40), (39) yields:

$$
|\chi|_{x y}^{2}(\nu, \tau)=\frac{1}{T_{c}} P_{x} P_{y} \gamma_{x y}(\nu, \tau) \text {. }
$$

The use of the WVS accounts for non-stationary random processes, whose spectra may change over time. However, if the random process $x$ is stationary (i.e. its statistical moments are constant over time), or at least quasi-stationary (i.e. its statistical moments are constant over a fraction of time $T_{c}$ ), the WVS becomes the stationary PSD [24]:

$$
W_{x}(t, f)=S_{x}(f),
$$

and, in this case, the GSSC yields:

$$
\begin{aligned}
\gamma_{x y}(\nu, \tau) & =\frac{1}{T_{c}} \overline{W_{x}}(\tau, \nu) * * \overline{W_{y}}(\tau, \nu) \\
& =\frac{1}{T_{c}} \iint_{T_{c}} \overline{W_{x}}(t, f) \overline{W_{y}}(t-\tau, f-\nu) d t d f \\
& =\frac{1}{T_{c}} \iint_{T_{c}} \overline{S_{x}}(f) \overline{S_{y}}(f-\nu) d t d f \\
& =\int \overline{S_{x}}(f) \overline{S_{y}}(f-\nu) d f .
\end{aligned}
$$

Therefore, in this case, the GSSC is equivalent to the classical definition of SSC in [31]:

$$
\gamma_{x y}(\nu, \tau)=\kappa_{x y}(\nu)=\overline{S_{x}}(\nu) * \overline{S_{y}}(\nu),
$$

and a CWAF can be expressed as:

$$
|\chi|_{x y}^{2}(\nu, \tau)=\frac{1}{T_{c}} P_{x} P_{y} \kappa_{x y}(\nu) .
$$

Furthermore, considering an ideal transfer function for the front-end with bandwidth $B_{r}$, the GSSC can be written as in (16), and the classical SSC turns to be equal to the one in (12).

\section{REFERENCES}

[1] "Power Utilities. Mitigating GPS Vulnerabilities and Protecting Power Utility Network Timing," Symmetricom Inc., San Jose, California, Technical Report, 2013. [Online]. Available: https://www.aventasinc. com/whitepapers/WP_Power_Utilities.pdf.[Lastaccessed]2016-07-26.

[2] J. Querol, G. F. Forte, and A. Camps, "Study of RFI signals in protected GNSS bands generated by common electronic devices: Effects on GNSS-R measurements," in 2014 IEEE Geoscience and Remote Sensing Symposium, Quebec, Canada, 2014, pp. 4050-4053.

[3] M. Wildemeersch, A. Rabbachin, E. Cano, and J. Fortuny, "Interference assessment of DVB-T within the GPS L1 and Galileo E1 band," in Programme and Abstract Book - 5th ESA Workshop on Satellite Navigation Technologies and European Workshop on GNSS Signals and Signal Processing, NAVITEC 2010, 2010.

[4] J. W. Betz, "Effect of Partial-Band Interference on Receiver Estimation of C/NO: Theory," The MITRE Corporation, Technical Report, pp. 716723, 2001.

[5] J. Betz and B. Titus, "Intersystem and intrasystem interference with signal imperfections," in PLANS 2004. Position Location and Navigation Symposium (IEEE Cat. No.04CH37556), 2004, pp. 558-565.

[6] C. Hall and R. Cordey, "Multistatic Scatterometry," in International Geoscience and Remote Sensing Symposium, 'Remote Sensing: Moving Toward the 21 st Century'., 1988, pp. 561-562.

[7] M. Martin-Neira, "A Passive Reflectometry and Interferometry System (PARIS) - Application to ocean altimetry," ESA journal, vol. 17, no. 4, pp. 331-355, 1993.
[8] A. Alonso-Arroyo, A. Camps, H. Park, D. Pascual, R. Onrubia, and F. Martin, "Retrieval of Significant Wave Height and Mean Sea Surface Level Using the GNSS-R Interference Pattern Technique: Results From a Three-Month Field Campaign," IEEE Transactions on Geoscience and Remote Sensing, vol. 53, no. 6, pp. 3198-3209, Jun. 2015.

[9] R. Sabia, M. Caparrini, and G. Ruffini, "Potential Synergetic Use of GNSS-R Signals to Improve the Sea-State Correction in the Sea Surface Salinity Estimation: Application to the SMOS Mission," IEEE Transactions on Geoscience and Remote Sensing, vol. 45, no. 7, pp. 2088-2097, Jul. 2007.

[10] N. Rodriguez-Alvarez, X. Bosch-Lluis, A. Camps, M. Vall-llossera, E. Valencia, J. Marchan-Hernandez, and I. Ramos-Perez, "Soil Moisture Retrieval Using GNSS-R Techniques: Experimental Results Over a Bare Soil Field," IEEE Transactions on Geoscience and Remote Sensing, vol. 47, no. 11, pp. 3616-3624, Nov. 2009.

[11] E. Cardellach, A. Rius, M. Martin-Neira, F. Fabra, O. Nogues-Correig, S. Ribo, J. Kainulainen, A. Camps, and S. D'Addio, "Consolidating the Precision of Interferometric GNSS-R Ocean Altimetry Using Airborne Experimental Data," IEEE Transactions on Geoscience and Remote Sensing, vol. 52, no. 8, pp. 4992-5004, Aug. 2014.

[12] A. W. Rihaczek, Principles of high-resolution radar. McGraw-Hill, 1969.

[13] V. U. Zavorotny, S. Gleason, E. Cardellach, and A. Camps, "Tutorial on Remote Sensing Using GNSS Bistatic Radar of Opportunity," IEEE Geoscience and Remote Sensing Magazine, vol. 2, no. 4, pp. 8-45, Dec. 2014.

[14] V. U. Zavorotny and A. G. Voronovich, "Scattering of GPS signals from the ocean with wind remote sensing application," IEEE Transactions on Geoscience and Remote Sensing, vol. 38, no. 2, pp. 951-964, 2000.

[15] T. Elfouhaily, D. R. Thompson, and L. Linstrom, "Delay-Doppler analysis of bistatically reflected signals from the ocean surface: Theory and application," IEEE Transactions on Geoscience and Remote Sensing, vol. 40 , no. 3 , pp. 560-573, 2002.

[16] J. F. Marchan-Hernandez, A. Camps, N. Rodriguez-Alvarez, E. Valencia, $\mathrm{X}$. Bosch-Lluis, and I. Ramos-Perez, "An efficient algorithm to the simulation of delay-Doppler maps of reflected global navigation satellite system signals," IEEE Transactions on Geoscience and Remote Sensing, vol. 47, no. 8, pp. 2733-2740, 2009.

[17] J. Ville, "Theorie et applications de la notion de signal analytique," Cables et Transmissions, vol. 2A, no. 1, pp. 61-74, 1948.

[18] P. Woodward, "Radar Ambiguity Analysis," R.R.E. Technical Note No 731, vol. February, 1967.

[19] E. Kaplan and C. Hegarty, Understanding GPS: Principles and Applications, Second Edition. Artech House, 2005.

[20] S. Sussman, "Least-square synthesis of radar ambiguity functions," IRE Transactions on Information Theory, vol. 8, no. 3, 1962.

[21] D. Pascual, H. Park, R. Onrubia, A. A. Arroyo, J. Querol, and A. Camps, "Crosstalk Statistics and Impact in Interferometric GNSSR," IEEE Journal of Selected Topics in Applied Earth Observations and Remote Sensing, pp. 1-10, 2016.

[22] M. Martín-Neira, S. D'Addio, C. Buck, N. Floury, and R. PrietoCerdeira, "The PARIS ocean altimeter in-Orbit demonstrator," IEEE Transactions on Geoscience and Remote Sensing, vol. 49, no. 6 PART 2, pp. 2209-2237, 2011.

[23] D. Pascual, A. Camps, F. Martin, H. Park, A. A. Arroyo, and R. Onrubia, "Precision bounds in GNSS-R ocean altimetry," IEEE Journal of Selected Topics in Applied Earth Observations and Remote Sensing, vol. 7, no. 5, pp. 1416-1423, 2014.

[24] W. Martin and P. Flandrin, "Wigner-Ville spectral analysis of nonstationary processes," IEEE Transactions on Acoustics, Speech, and Signal Processing, vol. 33, no. 6, 1985.

[25] J. Querol and A. Camps, "Sistema y método para la detección y eliminación en tiempo real de interferencias de radiofrecuencia," ES Patent, Application Submitted, 2015.

[26] R. Onrubia, J. Querol, D. Pascual, A. Alonso-Arroyo, H. Park, and A. Camps, "DME/TACAN Impact Analysis on GNSS Reflectometry," IEEE Journal of Selected Topics in Applied Earth Observations and Remote Sensing, pp. 1-10, 2016.

[27] J. Querol, A. Alonso-Arroyo, R. Onrubia, D. Pascual, and A. Camps, "Assessment of back-end RFI mitigation techniques in passive remote sensing," in 2015 IEEE International Geoscience and Remote Sensing Symposium (IGARSS). Milan, Italy: IEEE, Jul. 2015, pp. 4746-4749.

[28] L. Cohen, Time-frequency Analysis, ser. Electrical engineering signal processing. Prentice Hall PTR, 1995.

[29] W. M. Siebert, "Studies of Woodward's uncertainty function," MIT Quarterly Progress Report, pp. 90-94, 1958. 
[30] C. Stutt, "A note on invariant relations for ambiguity and distance functions," IEEE Transactions on Information Theory, vol. 5, no. 4, pp. 164-167, Dec. 1959.

[31] J. A. Avila-Rodríguez, "On Generalized Signal Waveforms for Satellite Navigation,” Ph.D. dissertation, University FAF Munich, 2008. 